УДК 517.95

N. P. Protsakh

\title{
PROBLEM OF DETERMINING OF MINOR COEFFICIENT AND RIGHT-HAND SIDE FUNCTION IN SEMILINEAR ULTRAPARABOLIC EQUATION
}

\begin{abstract}
N. P. Protsakh. Problem of determining of minor coefficient and right-hand side function in semilinear ultraparabolic equation, Mat. Stud. 50 (2018), 60-74.

The problem of determining of the right-hand side function and the time depended minor coefficient in semilinear ultraparabolic equation from the initial, boundary and overdetermination conditions, is considered in this paper. The sufficient conditions of the existence and the uniqueness of solution on some interval $[0, T]$, where $T$ depends on the coefficients of the equation, for the problem are obtained.
\end{abstract}

1. Introduction. The problems for ultraparabolic equations appear in mathematical modeling of many phenomena of mechanics, physics, biology and financial mathematics, for example, such as the diffusion with inertia, population dynamics, the theory of Asian options etc. [1]-[4].

The conditions of the unique solvability of Cauchy problems and the initial-boundary value problems for the ultraparabolic equations were investigated in the works [1]-[9], of the inverse problems of identifying of single or several unknown parameters in the right-hand side function of the semilinear ultraparabolic equations in [1, 10]--[13], of the inverse problem of identifying of a minor coefficient in the linear ultraparabolic equation in [14].

In the present paper, we consider the inverse problem for the semilinear ultraparabolic equation with the unknown minor coefficient and the time dependent parameter in the righthand side function of the equation. We set the boundary and the integral overdetermination conditions. With the use of Faedo-Galerkin method and the method of successive approximations we establish the sufficient conditions of the existence and the uniqueness of solutions from Sobolev spaces for the problem on some interval $[0, T]$.

Note, that the problems of determination of a single parameter in the right-hand side function or the minor coefficient of the parabolic equations were studied in [15]-[23], of the several coefficients were considered in [23]-[25]. The authors used the methods of the integral equations, regularization and the Shauder principle [14, 20, 21, 23, 24], the method of semigroups [22], the methods of finite difference approximations, numerical and iterative methods [15, 17, 18, 19, 25].

2. Statement of the problem. Let $\Omega \subset \mathbb{R}^{n}$ and $D \subset \mathbb{R}^{l}$ be bounded domains with the boundaries $\partial \Omega \in C^{2}$ and $\partial D \in C^{1} ; T \in(0, \infty), x \in \Omega, y \in D, t \in(0, T), G=\Omega \times D$, $Q_{\tau}=\Omega \times D \times(0, \tau), \tau \in(0, T], \Sigma_{T}=\partial \Omega \times D \times(0, T), S_{T}=\Omega \times \partial D \times(0, T), n, l \in \mathbb{N}$.

2010 Mathematics Subject Classification: 35K70, 35R30.

Keywords: inverse problem; ultraparabolic equation; boundary-value problem; unique solvability. doi:10.15330/ms.50.1.60-74

(C) N. P. Protsakh, 2018 
We shall use the spaces $L^{\infty}(\cdot), L^{2}(\cdot), W^{1,2}(\cdot), C^{k}(\cdot), C\left([0, T] ; L^{2}(G)\right), C^{1}\left(D ; C^{1}(\bar{\Omega})\right)$ from [26, pp. 32, 37, 38, 44, 147].

We consider the equation

$$
\begin{gathered}
u_{t}+\sum_{i=1}^{l} \lambda_{i}(x, y, t) u_{y_{i}}-\sum_{i, j=1}^{n}\left(a_{i j}(x, y, t) u_{x_{i}}\right)_{x_{j}}+(c(t)+b(x, y)) u+ \\
+g(x, y, t, u)=f_{1}(x, y, t) q(t)+f_{2}(x, y, t), \quad(x, y, t) \in Q_{T},
\end{gathered}
$$

with the initial condition

$$
u(x, y, 0)=u_{0}(x, y), \quad(x, y) \in G
$$

the boundary conditions

$$
\left.u\right|_{\Sigma_{T}}=0,\left.\quad u\right|_{S_{T}^{1}}=0
$$

and the overdetermination conditions

$$
\begin{array}{ll}
\int_{G} K_{1}(x, y) u(x, y, t) d x d y=E_{1}(t), & t \in[0, T], \\
\int_{G} K_{2}(x, y) u(x, y, t) d x d y=E_{2}(t), & t \in[0, T],
\end{array}
$$

where $u(x, y, t), c(t), q(t)$ are unknown functions,

$$
S_{T}^{1}:=\left\{(x, y, t) \in S_{T}: \sum_{i=1}^{l} \lambda_{i}(x, y, t) \cos \left(\nu, y_{i}\right)<0\right\}
$$

$\nu$ is the outward unit normal vector to $S_{T}$.

In this paper we shall study the following inverse problem: find the sufficient conditions of the existence and the uniqueness of a triple of functions $(u(x, y, t), c(t), q(t))$ such that the relations (1)-(5) hold in the sense of Definition 1 (see below).

Assume that

(S): there exists $\Gamma_{1} \subset \partial D \subset \mathbb{R}^{l-1}$, such that $S_{T}^{1}=\Omega \times \Gamma_{1} \times(0, T)$.

Denote $S_{T}^{2}:=\left\{(x, y, t) \in S_{T}: \sum_{i=1}^{l} \lambda_{i}(x, y, t) \cos \left(\nu, y_{i}\right) \geq 0\right\}, \Gamma_{2}=\partial D \backslash \Gamma_{1}$.

Assume that the following assumptions hold:
(A):

$$
a_{i j} \in C\left([0, T] ; L^{2}(G)\right), i, j=1, \ldots, n, \quad \sum_{i=1}^{n} a_{i j}(x, y, t) \xi_{i} \xi_{j} \geq a_{0}|\xi|^{2}
$$$$
\text { for almost all }(x, y, t) \in Q_{T} \text { and for all } \xi \in \mathbb{R}^{n}, a_{0}>0 \text {; }
$$
(L): $\quad \lambda_{i} \in C\left(\bar{Q}_{T}\right), \lambda_{i y_{i}} \in L^{\infty}\left(Q_{T}\right), \quad i=1, \ldots, l$;
(B): $\quad b \in L^{\infty}(G), b(x, y) \geq b_{0}$ for almost all $(x, y) \in G$, where $b_{0}$ is a constant;
(G): $\quad g(x, y, t, \xi)$ is measurable with respect to the variables $(x, y, t)$ in $Q_{T}$ for all $\xi \in \mathbb{R}^{1}$ and is continuous with respect to $\xi$ for almost all $(x, y, t) \in Q_{T}$, moreover, there exists a positive constant $g_{0}$, 
such that $|g(x, y, t, \xi)-g(x, y, t, \eta)| \leq g_{0}|\xi-\eta|$

for almost all $(x, y, t) \in Q_{T}$ and all $\xi, \eta \in \mathbb{R}^{1}$;

(F): $\quad f_{1}, f_{2} \in C\left([0, T] ; L^{2}(G)\right)$;

$(\mathbf{U}): \quad u_{0} \in W^{1,2}(G),\left.u_{0}\right|_{\partial \Omega \times D}=0,\left.u_{0}\right|_{\Omega \times \Gamma_{1}}=0$;

$(\mathbf{K}): \quad K_{i} \in C^{1}\left(D ; C^{1}(\bar{\Omega})\right),\left.K_{i}\right|_{\partial \Omega \times D}=0,\left.\quad K_{i}\right|_{\Omega \times \Gamma_{2}}=0, \quad i=1,2 ;$

$(\mathbf{E}): \quad E_{i} \in W^{1,2}(0, T), E_{i}(0)=\int_{G} K_{i}(x, y) u_{0}(x, y) d x d y, i=1,2$

3. Initial-boundary value (direct) problem. First we assume that in Eq. (1) $c(t)=c^{*}(t)$, $q(t)=q^{*}(t)$, where $c^{*} \in C([0, T]), q^{*} \in L^{2}(0, T)$, are known functions; consider the initialboundary value problem for the Eq. (1) with the initial condition (2) and with the boundary conditions (3).

We shall introduce the following spaces:

$$
\begin{aligned}
& V_{1}\left(Q_{T}\right):=\left\{w: w, w_{x_{i}} \in L^{2}\left(Q_{T}\right), i=1, \ldots, n,\left.w\right|_{\Sigma_{T}}=0\right\} ; \\
& V_{2}(G):=\left\{w: w \in W^{1,2}(G),\left.w\right|_{\partial \Omega \times D}=0,\left.w\right|_{\Omega \times \Gamma_{1}}=0\right\} ; \\
& V_{3}\left(Q_{T}\right):=\left\{w: w \in W^{1,2}\left(Q_{T}\right),\left.w\right|_{S_{T}^{1}}=0,\left.w\right|_{\Sigma_{T}}=0\right\} ; \\
& V_{4}\left(Q_{T}\right):=\left\{w: w \in V_{3}\left(Q_{T}\right), w_{x_{i} x_{j}} \in L^{2}\left(Q_{T}\right), i, j=1, \ldots, n\right\} .
\end{aligned}
$$

The results presented in [9] and [10] yield the following statements.

Theorem 1. Suppose that the conditions (A), (B), (G), (L), (F), (U), (S) hold, and, besides: 1) $a_{i j x_{i}}, a_{i j t} \in L^{\infty}\left(Q_{T}\right), b_{y_{k}} \in L^{\infty}\left(Q_{T}\right), f_{s y_{k}} \in L^{2}\left(Q_{T}\right), c^{*} \in C([0, T]), i, j=1, \ldots, n, k=$ $1, \ldots, l, s=1,2$;

2) there exists a constant $g_{1}$ such that for almost all $(x, y, t) \in Q_{T}$ and all $\xi \in \mathbb{R}^{1}$ the inequalities $\left|g_{y_{i}}(x, y, t, \xi)\right| \leq g_{1}, \quad i=1, \ldots, l$, are true and $\left.g(x, y, t, 0)\right|_{S_{T}^{1}}=0$;

3) $\left.f_{s}\right|_{S_{T}^{1}}=0, s=1,2$. Then there exists a unique function $u^{*} \in V_{3}\left(Q_{T}\right) \cap C\left([0, T] ; L^{2}(G)\right)$, that satisfies the condition (2) and the equality

$$
\begin{gathered}
\int_{Q_{T}}\left(u_{t}^{*} v+\sum_{i=1}^{l} \lambda_{i}(x, y, t) u_{y_{i}}^{*} v+\sum_{i, j=1}^{n} a_{i j}(x, y, t) u_{x_{i}}^{*} v_{x_{i}}+\left(c^{*}(t)+b(x, y)\right) u^{*} v+\right. \\
\left.\quad+g\left(x, y, t, u^{*}\right) v\right) d x d y d t=\int_{Q_{T}}\left(f_{1}(x, y, t) q^{*}(t)+f_{2}(x, y, t)\right) v d x d y d t
\end{gathered}
$$

for all functions $v \in V_{1}\left(Q_{T}\right)$. Moreover, $u^{*} \in V_{4}\left(Q_{T}\right) \cap C\left([0, T] ; L^{2}(G)\right)$, $u^{*}$ satisfies the condition (2) and Eq. (1) for almost all $(x, y, t) \in Q_{T}$ (so, $u^{*}$ is a solution to the problem $(1)-(3))$.

The proof is carried out according to the scheme of proving of Theorems 1, 2, Lemma 1 ([9]), Theorem 3 and Lemma 1 ([10]).

Remark 1. It follows from [9] that the derivatives of $u^{*}$ have the following estimates

$$
\int_{Q_{T}} \sum_{i=1}^{l}\left(u_{y_{i}}^{*}\right)^{2} d x d y d t \leq M_{0}, \quad \int_{Q_{T}}\left(u_{t}^{*}\right)^{2} d x d y d t \leq M,
$$

where the constants $M_{0}, M$ depend on $u_{0}$, and on the coefficients and the right-hand side function of Eq. (1). 


\section{Inverse problem.}

Definition 1. A triple of functions $(u(x, y, t), c(t), q(t))$ is a solution to the problem $(1)-(5)$, if $u \in V_{4}\left(Q_{T}\right) \cap C\left([0, T] ; L^{2}(G)\right), c \in C([0, T]), q \in L^{2}(0, T)$, it satisfies Eq. (1) for almost all $(x, y, t) \in Q_{T}$ and the conditions (2), (4), (5) hold.

Denote:

$$
\begin{gathered}
A_{i}(t):=-E_{i}^{\prime}(t)+\int_{G} K_{i}(x, y) f_{2}(x, y, t) d x d y, \quad i=1,2 \\
B_{s}(x, y, t):=\sum_{i=1}^{l}\left(\lambda_{i}(x, y, t) K_{s}(x, y)\right)_{y_{i}}+\sum_{i, j=1}^{l}\left(K_{s x_{j}}(x, y) a_{i j}(x, y, t)\right)_{x_{i}}-K_{s}(x, y) b(x, y), s=1,2, \\
F_{i}(t):=\int_{G} K_{i}(x, y) f_{1}(x, y, t) d x d y, \quad i=1,2 .
\end{gathered}
$$

Assume that

$$
E_{2}(t) F_{1}(t)-E_{1}(t) F_{2}(t) \neq 0 \text { for all } t \in[0, T]
$$

Denote

$$
\begin{aligned}
A_{3}(t) & :=\frac{A_{2}(t) F_{1}(t)-A_{1}(t) F_{2}(t)}{E_{2}(t) F_{1}(t)-E_{1}(t) F_{2}(t)}, \quad B_{3}(x, y, t):=\frac{B_{2}(x, y, t) F_{1}(t)-B_{1}(x, y, t) F_{2}(t)}{E_{2}(t) F_{1}(t)-E_{1}(t) F_{2}(t)}, \\
A_{4}(t) & :=\frac{A_{2}(t) E_{1}(t)-A_{1}(t) E_{2}(t)}{E_{2}(t) F_{1}(t)-E_{1}(t) F_{2}(t)}, \quad B_{4}(x, y, t):=\frac{B_{2}(x, y, t) E_{1}(t)-B_{1}(x, y, t) E_{2}(t)}{E_{2}(t) F_{1}(t)-E_{1}(t) F_{2}(t)}, \\
D_{1}(x, y, t) & :=\frac{K_{1}(x, y) F_{2}(t)-K_{2}(x, y) F_{1}(t)}{E_{2}(t) F_{1}(t)-E_{1}(t) F_{2}(t)}, D_{2}(x, y, t):=\frac{K_{2}(x, y) E_{1}(t)-K_{1}(x, y) E_{2}(t)}{E_{2}(t) F_{1}(t)-E_{1}(t) F_{2}(t)} .
\end{aligned}
$$

It follows from (1), (4) and (5) that the solution of the problem (1)-(5) satisfies the equalities

$$
\begin{aligned}
& c(t)=A_{3}(t)+\int_{G}\left(B_{3}(x, y, t) u+D_{1}(x, y, t) g(x, y, t, u)\right) d x d y, t \in[0, T], \\
& q(t)=A_{4}(t)+\int_{G}\left(B_{4}(x, y, t) u-D_{2}(x, y, t) g(x, y, t, u)\right) d x d y, t \in[0, T] .
\end{aligned}
$$

The way of deriving of (8) is shown in the proof of the necessity of Lemma 1.

Lemma 1. Let the assumptions of Theorem 1 and $(7),(K),(E)$ hold. The triple of functions $(u(x, y, t), c(t), q(t))$, where $u \in V_{4}\left(Q_{T}\right) \cap C\left([0, T] ; L^{2}(G)\right), c \in C([0, T]), q \in L^{2}([0, T])$, is a solution to the problem (1) - (5) if and only if it satisfies Eq. (1) for almost all $(x, y, t) \in Q_{T}$, and (2), (8) hold.

Proof. Necessity. Let $\left(u^{*}(x, y, t), c^{*}(t), q^{*}(t)\right)$ be a solution of problem (1)-(5). After differentiation (4) once with respect to $t$ we derive formulae

$$
\int_{G} K_{1}(x, y) u_{t}^{*}(x, y, t) d x d y=E_{1}^{\prime}(t), \quad t \in[0, T] .
$$


By using relations (1) and (9) we get

$$
\begin{aligned}
& \int_{G} K_{1}(x, y)\left(f_{1}(x, y, t) q^{*}(t)+f_{2}(x, y, t)-\sum_{i=1}^{l} \lambda_{i}(x, y, t) u_{y_{i}}^{*}-b(x, y) u^{*}+\right. \\
& \left.+\sum_{i, j=1}^{n}\left(a_{i j}(x, y, t) u_{x_{i}}^{*}\right)_{x_{j}}-c^{*}(t) u^{*}-g\left(x, y, t, u^{*}\right)\right) d x d y=E_{1}^{\prime}(t), t \in[0, T] .
\end{aligned}
$$

Integrating by parts in (10), in view of the condition (K), we obtain

$$
\begin{gathered}
-E_{1}(t) c^{*}(t)+F_{1}(t) q^{*}(t)+\int_{G}\left(K_{1}(x, y) f_{2}(x, y, t)+\right. \\
\left.+B_{1}(x, y, t) u^{*}-K_{1}(x, y) g\left(x, y, t, u^{*}\right)\right) d x d y=E_{1}^{\prime}(t), \quad t \in[0, T] .
\end{gathered}
$$

From (5) and (1) we get

$$
\begin{aligned}
& \int_{G} K_{2}(x, y)\left(f_{1}(x, y, t) q^{*}(t)+f_{2}(x, y, t)-\sum_{i=1}^{l} \lambda_{i}(x, y, t) u_{y_{i}}^{*}-b(x, y) u^{*}+\right. \\
& \left.+\sum_{i, j=1}^{n}\left(a_{i j}(x, y, t) u_{x_{i}}^{*}\right)_{x_{j}}-c^{*}(t) u^{*}-g\left(x, y, t, u^{*}\right)\right) d x d y=E_{2}^{\prime}(t), t \in[0, T]
\end{aligned}
$$

and

$$
\begin{gathered}
-E_{2}(t) c^{*}(t)+F_{2}(t) q^{*}(t)+\int_{G}\left(K_{2}(x, y) f_{2}(x, y, t)+\right. \\
\left.+B_{2}(x, y, t) u^{*}-K_{2}(x, y) g\left(x, y, t, u^{*}\right)\right) d x d y=E_{2}^{\prime}(t), \quad t \in[0, T] .
\end{gathered}
$$

Solving the system of equations (11), (13) with respect to $c^{*}(t)$ and $q^{*}(t)$ using the condition (7) we obtain that $\left(u^{*}(x, y, t), c^{*}(t), q^{*}(t)\right)$ satisfies (8). Moreover, $u^{*}$ satisfies the condition (2) and equality (1) for almost all $(x, y, t) \in Q_{T}$ with $c(t)=c^{*}(t), q(t)=q^{*}(t)$.

Sufficiency. Let $c^{*} \in C([0, T]), q^{*} \in L^{2}(0, T), u^{*} \in V_{4}\left(Q_{T}\right) \cap C\left([0, T] ; L^{2}(G)\right)$ and they satisfy (2), (8) and (1) for almost all $(x, y, t) \in Q_{T}$. Then $u^{*}$ is a solution to the problem (1)-(3) with $c^{*}$ and $q^{*}$ instead of $c$ and $q$ in Eq. (1).

We set $E_{i}^{*}(t)=\int_{G} K_{i}(x, y) u^{*}(x, y, t) d x d y, t \in[0, T], i=1,2$. In exactly the same way as in the proof of necessity, we obtain

$$
\begin{aligned}
& -F_{i}(t) q^{*}(t)+E_{i}^{*}(t) c^{*}(t)=-\left(E_{i}^{*}(t)\right)^{\prime}+\int_{G}\left(K_{i}(x, y) f_{2}(x, y, t)+\right. \\
& \left.+B_{i}(x, y, t) u^{*}-K_{i}(x, y) g\left(x, y, t, u^{*}\right)\right) d x d y, \quad t \in[0, T], i=1,2 .
\end{aligned}
$$

On the other hand $c^{*}(t), q^{*}(t)$ and $u^{*}(x, y, t)$ satisfy (8), and therefore it is easy to get the following equalities

$$
-F_{i}(t) q^{*}(t)+E_{i}(t) c^{*}(t)=-\left(E_{i}(t)\right)^{\prime}+\int_{G}\left(K_{i}(x, y) f_{2}(x, y, t)+\right.
$$




$$
\left.+B_{i}(x, y, t) u^{*}-K_{i}(x, y) g\left(x, y, t, u^{*}\right)\right) d x d y, \quad t \in[0, T], i=1,2
$$

It follows from (14), (15) that

$$
\left(E_{i}^{*}(t)-E_{i}(t)\right) c^{*}(t)=-\left(E_{i}^{*}(t)-E_{i}(t)\right)^{\prime}, t \in[0, T], i=1,2 .
$$

Integrating (16) with the use of the equality $E_{i}^{*}(0)=E_{i}(0)=\int_{G} K_{i}(x, y) u_{0}(x, y) d x d y$, we get $E_{i}^{*}(t)=E_{i}(t), t \in[0, T], i=1,2$, Hence, $u^{*}(x, y, t)$ satisfies the overdetermination conditions (4), (5). Lemma 1 is proved.

Denote: $\lambda^{1}=\max _{i} \operatorname{essup}_{Q_{T}}\left|\lambda_{i y_{i}}(x, y, t)\right| ; f_{3}=\sup _{[0, T] G}\left(f_{1}(x, y, t)\right)^{2} d x d y ; \gamma_{0}=\gamma_{0}(\Omega)$ is the coefficient in Friedrichs' inequality

$$
\begin{gathered}
\int_{\Omega}|v(x)|^{2} d x \leq \gamma_{0} \int_{\Omega} \sum_{i=1}^{n}\left|v_{x_{i}}(x)\right|^{2} d x, \quad v \in W_{0}^{1,2}(\Omega) ; \\
C_{1}:=3 \sup _{[0 ; T]}\left(\left(A_{3}(t)\right)^{2}+2\left(\int_{G} D_{1}(x, y, t) g(x, y, t, 0) d x d y\right)^{2}\right) ; \\
C_{2}:=3 \sup _{[0 ; T]}\left(\int_{G}\left(B_{3}(x, y, t)\right)^{2} d x d y+2\left(g_{0}\right)^{2} \int_{G}\left(D_{1}(x, y, t)\right)^{2} d x d y\right) ; \\
C_{3}:=3\left(\int_{0}^{T}\left(A_{4}(t)\right)^{2} d t+2 \int_{0}^{T}\left(\int_{G} D_{2}(x, y, t) g(x, y, t, 0) d x d y\right)^{2} d t\right) ; \\
C_{4}:=3 \sup _{[0 ; T]}\left(\int_{G}\left(B_{4}(x, y, t)\right)^{2} d x d y+2\left(g_{0}\right)^{2} \int_{G}\left(D_{2}(x, y, t)\right)^{2} d x d y\right) .
\end{gathered}
$$

Assume that there exist such numbers $T$ and $\delta$ that the following inequalities are true

$$
\begin{gathered}
f_{3} C_{4} T<\delta \\
\frac{2 a_{0}}{\gamma_{0}}+2 b_{0}-\lambda^{1} l-2 g_{0}-2 M_{2}-3 \delta>0
\end{gathered}
$$

where

$$
\begin{aligned}
& M_{1}:=\frac{1}{\delta} \int_{Q_{T}}\left(\left(f_{2}(x, y, t)\right)^{2}+(g(x, y, t, 0))^{2}\right) d x d y d t+\int_{G}\left(u_{0}(x, y)\right)^{2} d x d y \\
& M_{2}:=\left(C_{1}+C_{2} M_{1}+\frac{f_{3}}{\delta} C_{3} C_{2}+\frac{f_{3}}{\delta} C_{4} C_{2} M_{1} T+\frac{\left(\frac{f_{3}}{\delta}\right)^{2} C_{4} C_{2} T\left(C_{3}+C_{4} M_{1} T\right)}{1-\frac{f_{3}}{\delta} C_{4} T}\right)^{\frac{1}{2}} .
\end{aligned}
$$

Denote

$$
M_{3}:=C_{3}+C_{4} M_{1} T+\frac{f_{3}}{\delta} C_{3} C_{4} T+\frac{f_{3}}{\delta}\left(C_{4}\right)^{2} M_{1} T^{2}+\frac{\left(\frac{f_{3}}{\delta}\right)^{2} C_{4}^{2} T^{2}\left(C_{3}+C_{4} M_{1} T\right)}{1-\frac{f_{3}}{\delta} C_{4} T}
$$




$$
\begin{aligned}
M_{4} & :=M_{1}+\frac{f_{3}}{\delta} C_{3}+\frac{f_{3}}{\delta} C_{4} M_{1} T+\left(\frac{f_{3}}{\delta}\right)^{2} C_{3} C_{4} T+\left(\frac{f_{3}}{\delta}\right)^{2}\left(C_{4}\right)^{2} M_{1} T^{2}+ \\
& +\frac{\left(\frac{f_{3}}{\delta}\right)^{3}\left(C_{4}\right)^{2} T^{2}\left(C_{3}+C_{4} M_{1} T\right)}{1-\frac{f_{3}}{\delta} C_{4} T} ; \\
M_{5} & :=2\left(\int_{Q_{T}}\left(B_{3}(x, y, t)\right)^{2} d x d y d t+\left(g_{0}\right)^{2} \sup _{[0, T]} \int_{G}\left(D_{1}(x, y, t)\right)^{2} d x d y d t\right) ; \\
M_{6} & :=2\left(\int_{Q_{T}}\left(B_{4}(x, y, t)\right)^{2} d x d y d t+\left(g_{0}\right)^{2} \sup _{[0, T]} \int_{G}\left(D_{2}(x, y, t)\right)^{2} d x d y d t\right) ; \\
M_{7} & :=\max \left\{M_{1} ; f_{3}\right\} ; \\
M_{8} & :=\min \left\{T ; \frac{2 a_{0}}{\gamma_{0}}+2 b_{0}-\lambda^{1} l-2 g_{0}-2 M_{2}-2 \delta\right. \\
M_{9} & :=\left(M_{5}+M_{6}\right) M_{8} ; \\
M_{10} & :=2\left(\sup _{[0, T]} \int\left(B_{3}(x, y, t)\right)^{2} d x d y+\left(g_{0}\right)^{2} \sup _{[0, T]} \int_{G}\left(D_{1}(x, y, t)\right)^{2} d x d y\right) ; \\
M_{11} & :=\sqrt{\frac{M_{10} M_{7}}{\delta} .}
\end{aligned}
$$

Theorem 2. Let $M_{9}<1$, and let the hypotheses (7), (18), (19), (A), (B), (L), (U), (G), $(E),(K),(F),(S)$ hold, and $a_{i j x_{i}} \in L^{\infty}\left(Q_{T}\right), b_{y_{k}} \in L^{\infty}\left(Q_{T}\right), f_{s y_{k}} \in L^{2}\left(Q_{T}\right),\left.f_{s}\right|_{S_{T}^{1}}=0$, $i, j=1, \ldots, n, k=1, \ldots, l, s=1,2$. Then a solution to the problem (1)-(5) exists.

Proof. We use the method of successive approximations. We construct an approximation $\left(u^{m}(x, y, t), c^{m}(t), q^{m}(t)\right)$ to the solution of problem (1)-(5), where the functions $c^{m}(t)$ and $q^{m}(t), m \in \mathbb{N}$, satisfy the system of equalities

$$
\begin{gathered}
c^{1}(t):=0, q^{1}(t):=0, \\
c^{m}(t)=A_{3}(t)+\int_{G}\left(B_{3}(x, y, t) u^{m-1}+D_{1}(x, y, t) g\left(x, y, t, u^{m-1}\right)\right) d x d y, \\
t \in[0 ; T], m \geq 2, \\
q^{m}(t)=A_{4}(t)+\int_{G}\left(B_{4}(x, y, t) u^{m-1}-D_{2}(x, y, t) g\left(x, y, t, u^{m-1}\right)\right) d x d y, \\
t \in[0 ; T], m \geq 2,
\end{gathered}
$$

and $u^{m}$ satisfies the equality

$$
\int_{Q_{\tau}}\left(u_{t}^{m} v+\sum_{i=1}^{l} \lambda_{i}(x, y, t) u_{y_{i}}^{m} v+\sum_{i, j=1}^{n} a_{i j}(x, y, t) u_{x_{i}}^{m} v_{x_{j}}+\left(c^{m}(t)+b(x, y)\right) u^{m} v+\right.
$$


$\left.+g\left(x, y, t, u^{m}\right) v\right) d x d y d t=\int_{Q_{\tau}}\left(f_{1}(x, y, t) q^{m}(t)+f_{2}(x, y, t)\right) v d x d y d t, m \geq 1, \tau \in(0 ; T]$,

for all $v \in V_{1}\left(Q_{T}\right)$, and the condition

$$
u^{m}(x, y, 0)=u_{0}(x, y), \quad(x, y) \in G .
$$

It follows from Theorem 1 that for each $m \in \mathbb{N}$ there exists a unique function $u^{m} \in$ $V_{3}\left(Q_{T}\right) \cap C\left([0, T] ; L^{2}(G)\right)$, that satisfies (22), (23). Now we show that $c^{m}(t) \geq-M_{2}$ for all $m \in \mathbb{N}, t \in[0 ; T]$. Let $c^{m}(t) \geq c_{0 m}$ for all $t \in[0, T]$, where $c_{0 m} \in \mathbb{R}$. We shall find the estimate for $\int_{G}\left|u^{m}(x, y, \tau)\right|^{2} d x d y$. Let us choose $v=u^{m}$ in (22):

$$
\begin{gathered}
\int_{Q_{\tau}}\left(u_{t}^{m} u^{m}+\sum_{i=1}^{l} \lambda_{i}(x, y, t) u_{y_{i}}^{m} u^{m}+\sum_{i, j=1}^{n} a_{i j}(x, y, t) u_{x_{i}}^{m} u_{x_{j}}^{m}+\left(c^{m}(t)+b(x, y)\right)\left(u^{m}\right)^{2}+\right. \\
\left.+g\left(x, y, t, u^{m}\right) u^{m}\right) d x d y d t=\int_{Q_{\tau}}\left(f_{1}(x, y, t) q^{m}(t)+f_{2}(x, y, t)\right) u^{m} d x d y d t, \tau \in(0 ; T], m \geq 1 .
\end{gathered}
$$

Taking into account the hypotheses (A), (B), (L), (U), (G), (F), from (24) we obtain the inequalities

$$
\begin{aligned}
& \int_{G}\left(u^{m}(x, y, \tau)\right)^{2} d x d y+\int_{S_{\tau}^{2}} \sum_{i=1}^{l} \lambda_{i}(x, y, t)\left(u^{m}\right)^{2} \cos \left(\nu, y_{i}\right) d \sigma+2 a_{0} \int_{Q_{\tau}} \sum_{i=1}^{n}\left(u_{x_{i}}^{m}\right)^{2} d x d y d t+ \\
& \quad+\left(2 c_{0 m}-\lambda^{1} l+2 b_{0}-2 g_{0}-3 \delta\right) \int_{Q_{\tau}}\left(u^{m}\right)^{2} d x d y d t \leq \frac{1}{\delta} \int_{Q_{\tau}}\left(\left(f_{1}(x, y, t)\right)^{2}\left(q^{m}(t)\right)^{2}+\right. \\
& \left.+\left(f_{2}(x, y, t)\right)^{2}+(g(x, y, t, 0))^{2}\right) d x d y d t+\int_{G}\left(u_{0}(x, y)\right)^{2} d x d y, \tau \in(0 ; T], \quad m \geq 1 .
\end{aligned}
$$

After using the inequality (17) in the third term of (25), we get

$$
\begin{gathered}
\int_{G}\left(u^{m}(x, y, \tau)\right)^{2} d x d y+\int_{S_{\tau}^{2}} \sum_{i=1}^{l} \lambda_{i}(x, y, t)\left(u^{m}\right)^{2} \cos \left(\nu, y_{i}\right) d \sigma+ \\
+\left(\frac{2 a_{0}}{\gamma_{0}}-\lambda^{1} l+2 c_{0 m}+2 b_{0}-2 g_{0}-3 \delta\right) \int_{Q_{\tau}}\left(u^{m}\right)^{2} d x d y d t \leq \frac{1}{\delta} \int_{Q_{\tau}}\left(\left(f_{1}(x, y, t)\right)^{2}\left(q^{m}(t)\right)^{2}+\right. \\
\left.+\left(f_{2}(x, y, t)\right)^{2}+(g(x, y, t, 0))^{2}\right) d x d y d t+\int_{G}\left(u_{0}(x, y)\right)^{2} d x d y, \tau \in(0 ; T], \quad m \geq 1 .
\end{gathered}
$$

Using the assumption $\frac{2 a_{0}}{\gamma_{0}}-\lambda^{1} l+2 c_{0 m}+2 b_{0}-2 g_{0}-3 \delta \geq 0$, from (26) we get the estimates

$$
\int_{G}\left(u^{m}(x, y, \tau)\right)^{2} d x d y \leq M_{1}+\frac{1}{\delta} \int_{Q_{\tau}}\left|f_{1}(x, y, t)\right|^{2}\left(q^{m}(t)\right)^{2} d x d y d t, \tau \in(0 ; T], m \geq 1 .
$$


Rising up the both sides of Eq. (20) to the square and using the Hölder inequality, we get the estimate

$$
\left(c^{m}(t)\right)^{2} \leq C_{1}+C_{2} \int_{G}\left(u^{m-1}\right)^{2} d x d y, \quad t \in[0 ; T], m \geq 2 .
$$

Rising up the both sides of Eq. (21) to the square and using the Hölder inequality, after integrating with respect to $t$, we get the estimate

$$
\int_{0}^{T}\left(q^{m}(t)\right)^{2} d t \leq C_{3}+C_{4} \int_{Q_{T}}\left(u^{m-1}\right)^{2} d x d y d t, \quad m \geq 2 .
$$

It is easy to proof the estimates after using (27), (28), (29) and (18)

$$
\begin{gathered}
\left|c^{m}(t)\right| \leq M_{2}, \quad t \in[0, T], \quad m \geq 1, \\
\int_{0}^{T}\left(q^{m}(t)\right)^{2} d t \leq M_{3}, \quad m \geq 1, \\
\int_{G}\left(u^{m}(x, y, t)\right)^{2} d x d y \leq M_{4}, \quad t \in[0, T], m \geq 1 .
\end{gathered}
$$

Remark, that if we take $-M_{2}$ instead of $c_{0 m}$ and take into account the condition (19), we get

$$
\frac{2 a_{0}}{\gamma_{0}}-\lambda^{1} l+2 c_{0 m}+2 b_{0}-2 g_{0}-3 \delta=\frac{2 a_{0}}{\gamma_{0}}-\lambda^{1} l-2 M_{2}+2 b_{0}-2 g_{0}-3 \delta \geq 0 .
$$

Thus, for all $m \in \mathbb{N}: c^{m}(t) \geq-M_{2}$, and we can choose $c_{0 m}:=-M_{2}$ for all $m \in \mathbb{N}$.

Now we show that $\left\{\left(u^{m}(x, y, t), c^{m}(t), q^{m}(t)\right)\right\}_{m=1}^{\infty}$ converges to the solution of the problem (1)-(5). Denote

$$
\begin{gathered}
z^{m}:=z^{m}(x, y, t)=u^{m}(x, y, t)-u^{m-1}(x, y, t), \\
r^{m}(t):=c^{m}(t)-c^{m-1}(t), \quad s^{m}(t):=q^{m}(t)-q^{m-1}(t), \quad m \geq 2 .
\end{gathered}
$$

Formulas (20), (21) for $t \in[0, T]$ and $m \geq 2$ imply the equalities

$$
\begin{aligned}
& r^{m}(t)=\int_{G}\left(B_{3}(x, y, t) z^{m-1}+D_{1}(x, y, t)\left(\left(g\left(x, y, t, u^{m-1}\right)-g\left(x, y, t, u^{m-2}\right)\right)\right) d x d y,\right. \\
& s^{m}(t)=\int_{G}\left(B_{4}(x, y, t) z^{m-1}-D_{2}(x, y, t)\left(\left(g\left(x, y, t, u^{m-1}\right)-g\left(x, y, t, u^{m-2}\right)\right)\right) d x d y .\right.
\end{aligned}
$$

We square both sides of these equalities and integrate the result with respect to $t$, take into account that under the hypotheses $(\mathrm{G})$

$$
\int_{Q_{\tau}}\left(g\left(x, y, t, u^{m}\right)-g\left(x, y, t, u^{m-1}\right)\right) z^{m} d x d y d t \leq g_{0} \int_{Q_{\tau}}\left(z^{m}\right)^{2} d x d y d t, \quad \tau \in(0 ; T], m \geq 2,
$$


we obtain

$$
\begin{aligned}
& \int_{0}^{T}\left(r^{m}(t)\right)^{2} d t \leq M_{5} \int_{Q_{T}}\left(z^{m-1}\right)^{2} d x d y d t, \quad m \geq 2, \\
& \int_{0}^{T}\left(s^{m}(t)\right)^{2} d t \leq M_{6} \int_{Q_{T}}\left(z^{m-1}\right)^{2} d x d y d t, \quad m \geq 2 .
\end{aligned}
$$

It follows from $(23)$ that $z^{m}(x, y, 0)=0,(x, y) \in G, m \geq 2$. Hence, from (22), we get

$$
\begin{gathered}
\frac{1}{2} \int_{G}\left(z^{m}(x, y, \tau)\right)^{2} d x d y+\int_{Q_{\tau}}\left(\sum_{i=1}^{l} \lambda_{i}(x, y, t) z_{y_{i}}^{m} z^{m}+\sum_{i, j=1}^{n} a_{i j}(x, y, t) z_{x_{i}}^{m} z_{x_{j}}^{m}+b(x, y)\left(z^{m}\right)^{2}+\right. \\
\left.+\left(g\left(x, y, t, u^{m}\right)-g\left(x, y, t, u^{m-1}\right)\right) z^{m}+\left(c^{m}(t) u^{m}-c^{m-1}(t) u^{m-1}\right) z^{m}\right) d x d y d t= \\
=\int_{Q_{\tau}} f_{1}(x, y, t) s^{m}(t) z^{m} d x d y d t, \quad \tau \in(0 ; T], m \geq 2
\end{gathered}
$$

We note that $\left(c^{m}(t) u^{m}-c^{m-1}(t) u^{m-1}\right) z^{m}=c^{m}(t)\left(z^{m}\right)^{2}+r^{m}(t) u^{m-1} z^{m}$, and therefore

$$
\begin{gathered}
\int_{Q_{\tau}}\left(c^{m}(t) u^{m}-c^{m-1}(t) u^{m-1}\right) z^{m} d x d y d t \geq\left(-M_{2}-\frac{\delta}{2}\right) \int_{Q_{\tau}}\left(z^{m}\right)^{2} d x d y d t- \\
-\frac{1}{2 \delta} \int_{0}^{\tau}\left(r^{m}(t)\right)^{2}\left(\int_{G}\left(u^{m-1}(x, y, t)\right)^{2} d x d y\right) d t \geq\left(-M_{2}-\frac{\delta}{2}\right) \int_{Q_{\tau}}\left(z^{m}\right)^{2} d x d y d t- \\
-\frac{M_{1}}{2 \delta} \int_{0}^{\tau}\left(r^{m}(t)\right)^{2} d t, \quad \tau \in(0, T], m \geq 2 .
\end{gathered}
$$

The last term in (36)

$$
\int_{Q_{\tau}} f_{1}(x, y, t) s^{m}(t) z^{m} d x d y d t \leq \frac{\delta}{2} \int_{Q_{\tau}}\left(z^{m}\right)^{2} d x d y d t+\frac{f_{3}}{2 \delta} \int_{0}^{T}\left(s^{m}(t)\right)^{2} d t .
$$

Then, taking into account (A), (B), (L), (U), (G), (F) and (37), from (36) we get inequalities

$$
\begin{gathered}
\int_{G}\left(z^{m}(x, y, \tau)\right)^{2} d x d y+\int_{S_{\tau}^{2}} \sum_{i=1}^{l} \lambda_{i}(x, y, t)\left(z^{m}\right)^{2} \cos \left(\nu, y_{i}\right) d \sigma+ \\
+2 a_{0} \int_{Q_{\tau}} \sum_{i, j=1}^{n}\left(z_{x_{i}}^{m}\right)^{2} d x d y d t+\left(2 b_{0}-\lambda^{1} l-2 g_{0}-2 M_{2}-2 \delta\right) \int_{Q_{\tau}}\left(z^{m}\right)^{2} d x d y d t \leq \\
\leq \frac{M_{1}}{\delta} \int_{0}^{T}\left(r^{m}(t)\right)^{2} d t+\frac{f_{3}}{\delta} \int_{0}^{T}\left(s^{m}(t)\right)^{2} d t, \tau \in(0 ; T], m \geq 2 .
\end{gathered}
$$


After applying (17) to the third term of (38), we get the estimate

$$
\begin{gathered}
\int_{G}\left(z^{m}(x, y, \tau)\right)^{2} d x d y+\int_{S_{\tau}^{2}} \sum_{i=1}^{l} \lambda_{i}(x, y, t)\left(z^{m}\right)^{2} \cos \left(\nu, y_{i}\right) d \sigma+\left(2 b_{0}-l \lambda^{1}-2 g_{0}+\frac{2 a_{0}}{\gamma_{0}}-2 M_{2}-\right. \\
-2 \delta) \int_{Q_{\tau}}\left(z^{m}\right)^{2} d x d y d t \leq \frac{M_{1}}{\delta} \int_{0}^{T}\left(r^{m}(t)\right)^{2} d t+\frac{f_{3}}{\delta} \int_{0}^{T}\left(s^{m}(t)\right)^{2} d t, \tau \in(0 ; T], m \geq 2 .
\end{gathered}
$$

In view of the conditions (18), (19), from (39) we find the estimates

$$
\int_{G}\left(z^{m}(x, y, \tau)\right)^{2} d x d y \leq \frac{M_{7}}{\delta} \int_{0}^{T}\left(\left(r^{m}(t)\right)^{2}+\left(s^{m}(t)\right)^{2}\right) d t, \tau \in(0 ; T], m \geq 2,
$$

and

$$
\int_{Q_{T}}\left(z^{m}\right)^{2} d x d y d t \leq M_{8} \int_{0}^{T}\left(\left(r^{m}(t)\right)^{2}+\left(s^{m}(t)\right)^{2}\right) d t, \quad m \geq 2 .
$$

It follows from (34), (35) and (41) that

$$
\begin{gathered}
\int_{0}^{T}\left(\left(r^{m}(t)\right)^{2}+\left(s^{m}(t)\right)^{2}\right) d t \leq M_{9} \int_{0}^{T}\left(\left(r^{m-1}(t)\right)^{2}+\left(s^{m-1}(t)\right)^{2}\right) d t \leq \\
\leq\left(M_{9}\right)^{m-2} \int_{0}^{T}\left(\left(r^{2}(t)\right)^{2}+\left(s^{2}(t)\right)^{2}\right) d t, \quad m \geq 2 .
\end{gathered}
$$

It is easy to find the estimate

$$
\left(r^{m}(t)\right)^{2} \leq M_{10} \int_{G}\left(z^{m-1}(x, y, t)\right)^{2} d x d y, \quad t \in[0, T], m \geq 2,
$$

from (33). Further, with the use of (40), from (43) we get

$$
\left|r^{m}(t)\right| \leq M_{11}\left(\int_{0}^{T}\left(\left(r^{m-1}(t)\right)^{2}+\left(s^{m-1}(t)\right)^{2} d t\right)\right)^{\frac{1}{2}}, t \in[0, T], m \geq 2 .
$$

By using (42), (44) and the assumption $M_{9}<1$ we can show that the estimate

$$
\begin{gathered}
\left|c^{m+k}(t)-c^{m}(t)\right| \leq \sum_{i=m+1}^{m+k}\left|r^{i}(t)\right| \leq M_{11} \sum_{i=m+1}^{m+k}\left(\int_{0}^{T}\left(\left(r^{i-1}(t)\right)^{2}+\left(s^{i-1}(t)\right)^{2}\right) d t\right)^{\frac{1}{2}} \leq \\
\leq \sum_{i=m+1}^{m+k} M_{11}\left(M_{9}\right)^{\frac{i-3}{2}}\left(\int_{0}^{T}\left(\left(r^{2}(t)\right)^{2}+\left(s^{2}(t)\right)^{2}\right) d t\right)^{\frac{1}{2}} \leq
\end{gathered}
$$




$$
\begin{gathered}
\leq M_{11} \frac{\left(M_{9}\right)^{\frac{m-2}{2}}\left(1-\left(M_{9}\right)^{\frac{k}{2}}\right)}{1-\left(M_{9}\right)^{\frac{1}{2}}}\left(\int_{0}^{T}\left(\left(r^{2}(t)\right)^{2}+\left(s^{2}(t)\right)^{2}\right) d t\right)^{\frac{1}{2}} \leq \\
\leq M_{11} \frac{\left(M_{9}\right)^{\frac{m-2}{2}}}{1-\left(M_{9}\right)^{\frac{1}{2}}}\left(\int_{0}^{T}\left(\left(r^{2}(t)\right)^{2}+\left(s^{2}(t)\right)^{2}\right) d t\right)^{\frac{1}{2}},
\end{gathered}
$$

holds for all $k \in \mathbb{N}, m \geq 3$. Besides,

$$
\begin{gathered}
\left(\int_{0}^{T}\left(q^{m+k}(t)-q^{m}(t)\right) d t\right)^{\frac{1}{2}} \leq \sum_{i=m+1}^{m+k}\left(\int_{0}^{T}\left(\left(r^{i-1}(t)\right)^{2}+\left(s^{i-1}(t)\right)^{2}\right) d t\right)^{\frac{1}{2}} \leq \\
\leq \frac{\left(M_{9}\right)^{\frac{m-2}{2}}}{1-\left(M_{9}\right)^{\frac{1}{2}}}\left(\int_{0}^{T}\left(\left(r^{2}(t)\right)^{2}+\left(s^{2}(t)\right)^{2}\right) d t\right)^{\frac{1}{2}}, \quad k \in \mathbb{N}, m \geq 3 .
\end{gathered}
$$

It follows from (45), (46) that for any $\varepsilon>0$, there exists $m_{0}$ such that for all $k, m \in \mathbb{N}, m>$ $m_{0}$, the inequalities $\left\|c^{m+k}(t)-c^{m}(t) ; C([0, T])\right\| \leq \varepsilon$ and $\left\|q^{m+k}(t)-q^{m}(t) ; L^{2}(0, T)\right\| \leq \varepsilon$ are true. Hence, the sequence $\left\{c^{m}\right\}_{m=1}^{\infty}$ is fundamental in $C([0, T])$, and $\left\{q^{m}\right\}_{m=1}^{\infty}$ is fundamental in $L^{2}(0, T)$. Thus, it follows from (40) and (38) that $\left\{u^{m}\right\}_{m=1}^{\infty}$ is fundamental in $L^{2}\left(Q_{T}\right) \cap C\left([0, T] ; L^{2}(G)\right)$ and $\left\{u_{x_{i}}^{m}\right\}_{m=1}^{\infty}$ is fundamental in $L^{2}\left(Q_{T}\right)$ and, hence, as $m \rightarrow \infty$

$$
\begin{aligned}
u^{m} \rightarrow u \text { in } L^{2}\left(Q_{T}\right) & \cap C\left([0, T] ; L^{2}(G)\right), u_{x_{i}}^{m} \rightarrow u_{x_{i}} \text { in } L^{2}\left(Q_{T}\right), i=1, \ldots, n, \\
c^{m} & \rightarrow c \text { in } C([0, T]), \quad q^{m} \rightarrow q \text { in } L^{2}(0, T) .
\end{aligned}
$$

Remark 1 implies the following estimates

$$
\int_{Q_{T}} \sum_{i=1}^{l}\left(u_{y_{i}}^{m}\right)^{2} d x d y d t \leq M_{0}, \quad \int_{Q_{T}}\left(u_{t}^{m}\right)^{2} d x d y d t \leq M
$$

and, by virtue of the inequalities (30), (31), the constants $M_{0}, M$ are independent of $m$ and the estimates (48) are true for all $m \in \mathbb{N}$. In view of (48), we can select a subsequence of sequence $\left\{u^{m}\right\}_{m=1}^{\infty}$ (we preserve the same notation for this subsequence), such that

$$
\begin{gathered}
u_{y_{i}}^{m} \rightarrow u_{y_{i}} \text { weakly in } L^{2}\left(Q_{T}\right), i=1, \ldots, l, \\
u_{t}^{m} \rightarrow u_{t} \text { weakly in } L^{2}\left(Q_{T}\right)
\end{gathered}
$$

as $m \rightarrow \infty$. Taking into account (47), (49), from (20) and (21) we get that the triple of functions $(u(x, y, t), c(t), q(t))$ satisfies the system of equations $(6)$ and

$$
\begin{gathered}
\int_{Q_{\tau}}\left(u_{t} v+\sum_{i=1}^{l} \lambda_{i}(x, y, t) u_{y_{i}} v+\sum_{i, j=1}^{n} a_{i j}(x, y, t) u_{x_{i}} v_{x_{j}}+(c(t)+b(x, y)) u v+\right. \\
+g(x, y, t, u) v) d x d y d t=\int_{Q_{\tau}}\left(f_{1}(x, y, t) q(t)+f_{2}(x, y, t)\right) v d x d y d t
\end{gathered}
$$


for all $v \in V_{1}\left(Q_{T}\right), \tau \in(0 ; T]$. It follows from (50) that

$$
\begin{aligned}
\int_{\Omega}\left(u_{t} w\right. & +\sum_{i=1}^{l} \lambda_{i}(x, y, t) u_{y_{i}} w+\sum_{i, j=1}^{n} a_{i j}(x, y, t) u_{x_{i}} w_{x_{j}}+(c(t)+b(x, y)) u w+ \\
& +g(x, y, t, u) w) d x=\int_{\Omega}\left(f_{1}(x, y, t) q(t)+f_{2}(x, y, t)\right) w d x
\end{aligned}
$$

for almost all $(y, t) \in D \times(0 ; T)$ and for all $w \in W_{0}^{1,2}(\Omega)$. From (51) we derive that $u$ for almost all $(y, t) \in D \times(0 ; T)$ is a weak solution to the Dirichlet problem for the elliptic equation

$$
\begin{aligned}
\sum_{i, j=1}^{n}\left(a_{i j}(x, y, t) u_{x_{i}}\right)_{x_{j}} & =F(x, y, t), \quad x \in \Omega, \\
\left.u\right|_{\partial \Omega} & =0,
\end{aligned}
$$

where

$F(x, y, t)=f_{1}(x, y, t) q(t)+f_{2}(x, y, t)-u_{t}-\sum_{i=1}^{l} \lambda_{i}(x, y, t) u_{y_{i}}-(c(t)+b(x, y)) u-g(x, y, t, u)$.

Since condition (3) is satisfied and the function $F(\cdot, y, t) \in L^{2}(\Omega)$ for almost all $(y, t) \in D \times$ $(0 ; T)$, it follows from Theorem 7.3 in $[27$, p. 130], that there exists the unique weak solution $u$ of the problem $(52)-(53)$, and $u_{x_{i} x_{j}}(\cdot, y, t) \in L^{2}(\Omega)$, hence, $u(\cdot, y, t) \in W_{0}^{2,2}(\Omega)$ for almost all $(y, t) \in D \times(0 ; T)$. Hence, $u \in V_{4}\left(Q_{T}\right) \cap C\left([0, T] ; L^{2}(G)\right)$, the triple $(u(x, y, t), c(t), q(t))$ satisfies Eq. (1) for almost all $(x, y, t) \in Q_{T}$, and by virtue of Lemma $1(u(x, y, t), c(t), q(t))$ is a solution of the problem $(1)-(5)$ in $Q_{T}$.

Theorem 3. Assume that the hypotheses of Theorem 2 are satisfied. Then a solution of the problem (1)-(5) is unique.

Proof. Assume that $\left(u_{(1)}(x, y, t), c_{(1)}(t), q_{(1)}(t)\right)$ and $\left(u_{(2)}(x, y, t), c_{(2)}(t), q_{(2)}(t)\right)$ are two solutions of problem (1)-(5). Then the triple of functions $(\tilde{u}(x, y, t), \tilde{c}(t), \tilde{q}(t))$, where $\tilde{u}(x, y, t)=u_{(1)}(x, y, t)-u_{(2)}(x, y, t), \tilde{c}(t)=c_{(1)}(t)-c_{(2)}(t), \tilde{q}(t)=q_{(1)}(t)-q_{(2)}(t)$, satisfies the condition $\tilde{u}(x, y, 0) \equiv 0$, the equality

$$
\begin{aligned}
& \int_{Q_{\tau}}\left(\tilde{u}_{t} v+\sum_{i=1}^{l} \lambda_{i}(x, y, t) \tilde{u}_{y_{i}} v+\sum_{i, j=1}^{n} a_{i j}(x, y, t) \tilde{u}_{x_{i}} v_{x_{j}}+b(x, y) \tilde{u} v+\left(c_{(1)}(t) u_{(1)}-c_{(2)}(t) u_{(2)}\right) v+\right. \\
& \left.\quad+\left(g\left(x, y, t, u_{(1)}\right)-g\left(x, y, t, u_{(2)}\right)\right) v\right) d x d y d t=\int_{Q_{\tau}} f_{1}(x, y, t) \tilde{q}(t) v d x d y d t, \tau \in[0, T], \quad(54)
\end{aligned}
$$

for all $v \in V_{1}\left(Q_{T}\right)$ and the system of equalities

$$
\tilde{c}(t)=\int_{G}\left(B_{3}(x, y, t) \tilde{u}+D_{1}(x, y, t)\left(\left(g\left(x, y, t, u_{(1)}\right)-g\left(x, y, t, u_{(2)}\right)\right)\right) d x d y\right.
$$




$$
\tilde{q}(t)=\int_{G}\left(B_{4}(x, y, t) \tilde{u}-D_{2}(x, y, t)\left(\left(g\left(x, y, t, u_{(1)}\right)-g\left(x, y, t, u_{(2)}\right)\right)\right) d x d y, t \in[0, T]\right.
$$

holds. After choosing $v=\tilde{u}$, in (54) we get

$$
\begin{aligned}
& \int_{Q_{\tau}}\left(\tilde{u}_{t} \tilde{u}+\sum_{i=1}^{l} \lambda_{i}(x, y, t) \tilde{u}_{y_{i}} \tilde{u}+\sum_{i, j=1}^{n} a_{i j}(x, y, t) \tilde{u}_{x_{i}} \tilde{u}_{x_{j}}+\left(c_{(1)}(t) u_{(1)}-c_{(2)}(t) u_{(2)}\right) \tilde{u}+b(x, y)(\tilde{u})^{2}+\right. \\
& \left.\quad+\left(g\left(x, y, t, u_{(1)}\right)-g\left(x, y, t, u_{(2)}\right)\right) \tilde{u}\right) d x d y d t=\int_{Q_{\tau}} f_{1}(x, y, t) \tilde{q}(t) \tilde{u} d x d y d t, \tau \in(0 ; T] . \quad(56)
\end{aligned}
$$

It is easy to get from $(55)$ and $(\mathrm{G})$ inequalities

$$
\int_{0}^{T}(\tilde{c}(t))^{2} d t \leq M_{5} \int_{Q_{T}}(\tilde{u})^{2} d x d y d t, \quad \int_{0}^{T}(\tilde{q}(t))^{2} d t \leq M_{6} \int_{Q_{T}}(\tilde{u})^{2} d x d y d t,
$$

From (56) by the same way as from (36) we got (41), we find the following estimate:

$$
\int_{Q_{T}}(\tilde{u})^{2} d x d y d t \leq M_{8} \int_{0}^{T}\left((\tilde{c}(t))^{2}+(\tilde{q}(t))^{2}\right) d t
$$

and taking into account (57) from (58), we obtain $\left(1-M_{9}\right) \int_{Q_{T}}(\tilde{u})^{2} d x d y d t \leq 0$. Since $M_{9}<1$, we conclude that $\int_{Q_{T}}(\tilde{u})^{2} d x d y d t=0$, hence, $u_{(1)}=u_{(2)}$ in $Q_{T}$. Then (57) implies $\tilde{c}(t) \equiv 0, \tilde{q}(t) \equiv 0$, and, therefore, $c_{(1)}(t) \equiv c_{(2)}(t), q_{(1)}(t) \equiv q_{(2)}(t)$ in $Q_{T}$.

5. Conclusion. In this paper on some time interval we have obtained the sufficient conditions of the existence and the uniqueness of the solution for the inverse problem for semilinear ultraparabolic equation with the unknown time dependent functions in the minor coefficient and in the right-hand side function of the equation. The next step of investigation of the problem is to get similar results when the condition (7) is not fulfilled and to prove the existence of the global solution for the problem.

\section{REFERENCES}

1. N.P. Protsakh, B.Yo. Ptashnyk, Nonlinear ultraparabolic equations and variational inequalities, "Naukova dumka", Kyiv, 2017, 278 p. (in Ukrainian)

2. S.D. Eidelman, S.D. Ivasyshen, A.N. Kochubei, Analytic methods in the theory of differential and pseudo-differential equations of parabolic type, Birkhäuser Verlag, 2004, 390 p.

3. A.N. Kolmogorov, Zufällige Bewegungen (Zur Theorie der Brownschen Bewegung), Ann. Math., 35 (1934), 116-117.

4. E. Lanconelli, A. Pascucci, S. Polidoro, Linear and nonlinear ultraparabolic equations of Kolmogorov type arising in diffusion theory and in finance, Nonlinear problems in mathematical physics and related topics II. In honour of Prof. O.A. Ladyzhenskaya. New York, NY: Kluwer Academic Publishers. Int. Math. Ser., N.Y., 2002, 243-265. 
5. S.D. Ivasishen, I.P. Medynsky, The Fokker-Planck-Kolmogorov equations for some degenerate diffusion processes, Theory Stoch. Process., 16(32) (2010), №1, 57-66.

6. G. Avalishvili, M. Avalishvili, Nonclassical problem for ultraparabolic equation in abstract spaces, Journal of Function Spaces, 2016 (2016), 1-15.

7. A.S. Tersenov, On the global solvability of the Cauchy problem for a quasilinear ultraparabolic equation, Asymptotic Analysis, 82 (2013), №3-4, 295-314.

8. S. Lavrenyuk, N. Protsakh, Boundary value problem for nonlinear ultraparabolic equation in unbounded with respect to time variable domain, Tatra Mt. Math. Publ., 38 (2007), 131-146.

9. S.P. Lavrenyuk, N.P. Protsakh, Mixed problem for a nonlinear ultraparabolic equation that generalizes the diffusion equation with inertia, Ukrainian Mathematical Journal, 58 (2006), №9, 1347-1368.

10. N. Protsakh, Inverse problem for a weakly nonlinear ultraparabolic equation with three unknown functions of different arguments on the right-hand side, Journal of Mathematical Sciences, 217 (2016), №4, 476514.

11. N. Protsakh, Determining of right-hand side of higher order ultraparabolic equation, Open Math., 15 (2017), 1048-1062.

12. N. Protsakh, Inverse problem for an ultraparabolic equation with unknown function of the space variable on the right-hand side, Journal of Mathematical Sciences, 203 (2014), №1, 16-39.

13. N. Protsakh, Inverse problem for semilinear ultraparabolic equation of higher order, Mathematica Bohemica, 140 (2015), №4, 335-404.

14. Yu.A. Kosheleva, On the solvability of the nonlinear inverse problems for ultraparabolic equations, Sib. Elektron. Mat. Izv., 13 (2016), 1187-1206.

15. M. Dehghan, Determination of an unknown parameter in a semi-linear parabolic equation, Mathematical problems in engineering, 8 (2002), №2, 111-122.

16. J.R. Cannon, Y. Lin, Determination of a parameter $p(t)$ in some quasi-linear parabolic differential equations, Inverse Probl., 4 (1988), 35-45.

17. F. Parzilvand, A.M. Shahrezaee, An inverse problem of identifying the coefficient of semilinear parabolic equation, Mathematical Researches (Sci. Kharazmi University), 2 (2016), №1, 79-88.

18. V.T. Borukhov, G.M. Zayats, Identification of a time-dependent source term in nonlinear hyperbolic or parabolic heat equation, International Journal of Heat and Mass Transfer, 91 (2015), 1106-1113.

19. P.N. Vabishchevich, V.I. Vasil'ev, Numerical solving the identifcation problem for the lower coencient of parabolic equation, arXiv:1304.5923v1 [cs.NA] 22 Apr 2013, 12p.

20. A.I. Prilepko, V.L. Kamynin, A.B. Kostin, Inverse source problem for parabolic equation with the condition of integral observation in time, Computational Mathematics and Mathematical Physics, 57 (2017), №6, 956-966.

21. H.P. Lopushanska, Determination of a minor coefficient in a time fractional diffusion equation, Mat. Stud., 45 (2016), №1, 57-66.

22. E. Ozbilge, A. Demir, Identification of the unknown diffusion coefficient in a linear parabolic equation via semigroup approach, Advances in Difference Equations, 2014 (2014), №47, 1-8.

23. M.I. Ivanchov, Inverse problems for equations of parabolic type, 238 pp., Mathematical Studies, Monograph Series, VNTL Publishers., V.10, 2003.

24. A.I. Kozhanov, An inverse problem with an unknown coefficient and right-hand side for a parabolic equation II, J. of Inverse and ill posed problems,11 (2003), №5, 505-522.

25. S.G. Pyatkov, E.I. Safonov, Determination of the source function in mathematical models of convectiondiffusion, Mat. Zametki Severo-Vost. Fed. Univ., 21 (2014), №2, 117-130.

26. H. Gajewski, K. Gröger, K. Zacharias, Nichtlineare Operatorgleichungen und Operatordifferentialgleichungen. Berlin, Akademie-Verlag, 1974.

27. O.A. Ladyzhenskaya, Boundary-value problems of mathematical physics, Nauka, Moscow, 1973,407 p. (in Russian)

National Forestry Engineering University of Ukraine, Lviv, Ukraine protsakh@ukr.net 